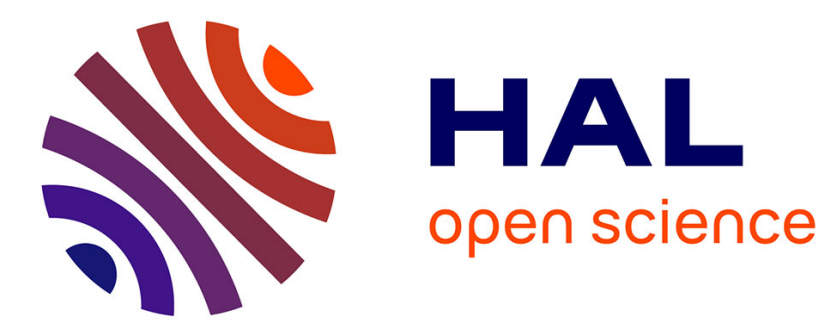

\title{
L'image du magasin : définition, effets, mesure et perspectives de recherche
}

Christophe Bezes

\section{To cite this version:}

Christophe Bezes. L'image du magasin : définition, effets, mesure et perspectives de recherche. Revue management \& avenir, 2013, 60 (2), pp.74-101. 10.3917/mav.060.0074 . hal-02086704

\section{HAL Id: hal-02086704 https://hal.science/hal-02086704}

Submitted on 1 Apr 2019

HAL is a multi-disciplinary open access archive for the deposit and dissemination of scientific research documents, whether they are published or not. The documents may come from teaching and research institutions in France or abroad, or from public or private research centers.
L'archive ouverte pluridisciplinaire HAL, est destinée au dépôt et à la diffusion de documents scientifiques de niveau recherche, publiés ou non, émanant des établissements d'enseignement et de recherche français ou étrangers, des laboratoires publics ou privés. 


\title{
L'image du magasin : définition, effets, mesure et perspectives de recherche ${ }^{1}$
}

\author{
Christophe Bèzes \\ Professeur-chercheur Istec* \\ Chercheur associé Largepa - Université Panthéon-Assas \\ c.bezes@istec.fr \\ * Istec, 12 rue Alexandre Parodi 75010 Paris
}

${ }^{1}$ Cet article a été initié par un cahier de recherche Istec intitulé «L'image du magasin : un état de l'art ». 


\title{
L'image du magasin : définition, effets, mesure et perspectives de recherche
}

\section{Résumé :}

Les magasins peuvent être analysés en mobilisant des concepts qui paraissent souvent interchangeables : image, qualité, atmosphère, personnalité ou expérience. Cette revue de littérature montre que ces concepts sont liés mais bien distincts. L'étude d'image semble plus particulièrement appropriée pour expliquer la sélection d'un magasin, dans le cadre d'achats planifiés et à partir de critères essentiellement cognitifs.

Mots-clés : magasin, image, qualité, atmosphère, personnalité, expérience.

\section{The store image: definition, effects, measurement and research perspectives}

\begin{abstract}
:
Stores can be analyzed by mobilizing concepts that often seem interchangeable: quality, atmosphere, personality, image or experience. This literature review shows that these concepts are related but distinct. The image study seems particularly appropriate to explain store selection, for planned purchases, using cognitive criteria.
\end{abstract}

Key words: store, image, quality, atmosphere, personality, experience. 
Les recherches sur le processus de décision des consommateurs se sont longtemps focalisées sur le choix du produit ou de la marque, sans tenir compte de la sélection de son lieu d'achat. Or cette conception feint d'ignorer la volonté de certains d'entre eux de regrouper leurs achats sous un même toit par souci de commodité et d'économie financière ou cognitive (AntéblianLambrey et François, 1998). C'est pourquoi Lusch (1982) ${ }^{2}$ a proposé différents scénarios selon que le consommateur choisit à la fois le produit et son lieu d'achat, le produit puis le lieu d'achat, ou bien l'inverse.

Particulièrement dans ce dernier cas, l'image du point de vente est importante car elle doit créer la confiance autour du produit finalement acheté. Par exemple, la taille des magasins conditionne souvent l'image-prix, ce qui peut réduire l'énergie consacrée à la recherche d'informations, une source apparaissant supérieure aux autres (Duncan et Olshavsky, 1982). C'est notamment vrai pour les services (restaurants, agences bancaires) où l'image de la surface de vente agit comme un indicateur tangible (Ward et al., 1992).

Plus généralement, l'image des magasins est réputée constituer le fondement du positionnement et de l'image de l'enseigne (Ailawadi et Keller, 2004). C'est ainsi que la dégradation actuelle du positionnement concurrentiel de Tesco sur son marché domestique semble être imputable au fait d'avoir trop privilégié dans ses magasins, un discours prix au détriment du choix, des services et de l'agencement ${ }^{3}$.

Cependant, malgré sa capacité à balayer l'ensemble des variables du retailing mix, le concept d'image du magasin tend à perdre sa prédominance initiale au profit de concepts connexes tels que l'atmosphère ou l'expérience de magasinage; nombre d'analystes confondent d'ailleurs ces concepts. Cette revue de littérature poursuit donc trois objectifs : 1) mieux délimiter le périmètre de l'image du magasin par rapport aux concepts apparentés d'atmosphère, de personnalité, de qualité ou d'expérience ; 2) examiner ses effets démontrés sur les comportements des consommateurs ; 3) faire le point sur sa mesure.

Après quelques généralités sur le concept d'image, nous aborderons ces différents points, en proposant pour chacun d'entre eux des voies de recherche.

\section{Généralités sur l'image}

Selon qu'elle est graphique, optique, perceptive, mentale ou verbale, l'image recouvre des notions extrêmement hétérogènes. Avant d'être réifiée par le monde occidental, elle fut d'abord pour Mitchell (1984, p.521) «abstraite, générale et spirituelle », en référence à Dieu. Plus prosaïquement, le marketing applique ce concept au produit, à la marque, à l'institution, au soi ou au magasin. Des images accumulées tout au long de sa vie, dépendrait en effet le comportement de tout individu (Enis 1967, se référant à Boulding).

L'image est une «représentation mentale d'origine sensible» (Petit Robert), qui met en relation «l'ensemble des perceptions sensorielles et des pensées associées par un individu à une entité » (Enis 1967, p.51) ; elle «ne décrit pas seulement des traits ou des qualités individuelles, mais l'impression totale que fait une entité dans l'esprit des autres... une image n'est pas simplement ancrée dans les données et les détails objectifs. C'est la configuration du plein champ de l'objet » (Dichter 1985, p.75).

Fondée sur ce que l'individu a expérimenté directement ou indirectement (lecture, photographie, conversations), elle constitue «une abstraction, une simplification de la réalité » (Enis 1967, p.51). Son processus de formation est en effet un phénomène séquentiel

\footnotetext{
2 Cité par Antéblian-Lambrey et François (1998).

3 The Grocer du 14 janvier 2012.
} 
et «subjectif qui prend place dans la réalité perçue » (Mazursky et Jacoby 1986, p.148) : «le client extrait et perçoit certains traits de la réalité (en partie contrôlés par le détaillant) et se façonne des croyances et/ou des affects congruents avec sa configuration cognitive idiosyncrasique » (Mazursky et Jacoby 1986, p.148).

L'image est également définie comme «la représentation psychique d'un objet absent» (Ingarao 2004, p.44) : «quand le client pense au magasin, quelques dimensions les plus saillantes sont retrouvées par la mémoire pour représenter ce que le magasin signifie » (Mazursky et Jacoby 1986, p. 148). C'est pourquoi Buttle (1985) envisage la possibilité d'une scission dans le temps et dans l'espace entre l'objet considéré et la perception qu'en ont les individus. En cela, il rejoint la distinction opérée par Gavard-Perret (1987, p.49) entre «l'image externe, stimulus visuel (tel qu'un dessin, une photographie) et l'image mentale reposant, elle, sur des images internes qui sont la reproduction ou la représentation mentale d'une perception ou d'une impression antérieure, en l'absence de l'objet (chose, relation, concept, etc.) lui ayant donné naissance ».

En marketing, Mazursky et Jacoby (1986, p.147) synthétisent ces deux facettes de l'image en la définissant comme «une connaissance et/ou un sentiment (ou un ensemble de connaissances et/ou de sentiments) qui est inféré d'un ensemble de perceptions en cours et/ou de souvenirs attachés à un phénomène (un objet ou un événement comme un magasin, un produit, une vente,...) et qui représente ce que le phénomène signifie pour l'individu ».

Plusieurs théories psychologiques ou économiques expliquent la construction de l'image et son impact sur les individus (Baker et al., 2002).

Selon la théorie des affordances de Gibson, «les individus perçoivent leur environnement physique comme une entité signifiante » (Baker et al. 2002, p.122). Cette théorie distingue les affordances réelles qui renvoient à ce qu'un produit offre et les affordances perçues qui correspondent à ce que chaque client pense que le produit est en mesure de lui offrir.

De son côté, la théorie des inférences considère que « le consommateur évalue un produit sur la base de ses attributs visibles (accessibles), apprête cette information via une ou plusieurs règles, et forme une préférence ou fait un choix » (Huber et Mc Cann 1982, p.324). L'absence d'un attribut entravant la décision d'achat, peut être compensée par le choix d'un processus d'inférence: assignation d'une valeur moyenne aux éléments manquants sur la base d'informations extérieurement disponibles ou déjà mémorisées (Dick et al., 1990), liaison d'un trait individuel à une impression plus générale (théorie du halo), ou création d'un lien de causalité, par exemple entre la qualité et le prix (théorie du signal).

Cette dernière théorie est sous-tendue par la notion d'asymétrie informationnelle. Elle pose qu'une partie (la firme), détentrice d'informations, va choisir de révéler à l'autre partie (le consommateur), dépourvue de compétences pour les évaluer directement, des indices ou indicateurs: le signal. Cette stratégie est la plus efficace en phase de préachat, lorsque l'information est rare (cas des produits nouveaux ou d'expérience), à condition que «les informations sur la qualité après l'achat soient sans équivoque, les acteurs informés des conséquences pour les deux parties et l'obligation soumise à la sanction du grand public » (Kirmani et Rao 2000, p.73).

Dans tous les cas, le choix d'un processus d'inférence affecte le résultat final (Dick et al., 1990)

Dans les faits, l'image se décompose en image voulue, image transmise et image perçue ou déposée (Marion, 1989): la première correspond au positionnement que l'entreprise souhaiterait transmettre à son marché ; la deuxième est la traduction de cette image voulue dans l'ensemble des supports de communication matériels ou immatériels, humains ou 
technologiques de l'entreprise; la dernière est «la construction forgée par un groupe d'individus lorsque ceux-ci partagent un processus commun de représentation » (Marion 1989, p.23), cette notion de représentation cherchant à combler ce qui sépare le sensible de l'intelligible. Un décalage apparaît presque toujours entre ces trois images qui devraient en principe être identiques (Birtwistle et al., 1999; Rattier, 2002). L'image est en effet subjective, multiple et réductrice (Pontier, 1988) :

- subjective car elle varie d'un individu à l'autre selon «ses expériences passées, son système de valeur, son état mental et physique au moment de la perception, les actions des autres individus et ses espérances pour l'avenir, avec des paramètres tels que son environnement culturel ou technologique et la structure de sa langue » (Enis 1967, p.55). L'image des magasins varie ainsi selon l'âge des personnes interrogées (Joyce et Lambert, 1996), les attitudes de chaque individu (structures de référence, concordance avec l'image de soi) ou leur rapport à l'objet analysé : les clients actuels, les anciens clients, les vendeurs et les managers ne perçoivent pas le magasin de la même manière (Samli et al., 1998 ; Keaveney et Hunt, 1992) ;

- multiple car elle combine des éléments rationnels et affectifs ;

- réductrice car «la réalité est différente de la perception» (Perrien et al., 1984) ${ }^{4}$. Ne pouvant saisir toute la complexité «des situations et des stimuli » (Lindquist 1974, p.29), l'esprit humain simplifie sa perception en se raccrochant aux seules significations qui lui paraissent saillantes.

Plus la zone de chevauchement entre chacune de ces perceptions est large (ce que Boulding nomme «image publique »), plus les différents acteurs ont la capacité de communiquer autour d'une même entité. Lazer et Wyckham (1969) montrent que cette communauté de perceptions existe aussi dans l'image des magasins, ce qui remet en partie en cause la vision segmentée en classes sociales de Martineau (1958).

\section{L’image du magasin : délimitation du concept}

Inspirée de celle du produit, l'image du magasin est l'un des plus anciens concepts utilisés par la recherche en distribution : il daterait de Greyser en 1936. Parmi les centaines de travaux qui l'ont abordé, Jallais et al. (1987, p.152) identifient deux grands courants de recherche : l'un conceptuel, «cherche à identifier les éléments constitutifs de l'image de magasin et à fonder l'existence même du concept»; l'autre «plus empirique » met l'accent sur des écarts d'image « significatifs » entre magasins, et déduit un lien avec leur fréquentation. L'image est en effet, aussi utile pour jauger les forces et les faiblesses de son point de vente face à ses concurrents, que pour prédire le choix de ceux qui les fréquentent (Wu et Petroshius, 1987).

Après avoir défini l'image du magasin, nous tenterons de mettre en évidence ses spécificités par rapport aux concepts apparemment proches de personnalité, d'atmosphère, de qualité et d'expérience.

\subsection{Définition du concept d'image du magasin}

La plupart des auteurs s'accordent à penser qu'en parlant de "personnalité du magasin », Martineau a en fait, livré la première définition de son image (d'Astous et al., 2002; Ambroise et al., 2003). Il la conçoit comme « la façon dont un magasin est défini dans l'esprit du consommateur en partie par ses qualités fonctionnelles et en partie par une aura d'attributs psychologiques » (Martineau 1958, p.47). L'apport de Martineau est d'autant plus remarquable qu'il énonce deux idées particulièrement novatrices : (1) l'influence de l'image

\footnotetext{
${ }^{4}$ Cités par Colombel et al. (2007, p.7).
} 
du magasin sera d'autant plus puissante que l'enseigne cherchera à compenser la disparition des contacts personnels, ce qui prend une autre valeur à l'ère du e-commerce ; (2) le shopping est pensé par les consommateurs «comme une expérience totale» (Martineau 1958, p.55). En effet, le chaland n'étant pas uniquement sensible à la communication sur les prix et aux économies à réaliser, Martineau insiste sur le rôle joué par les éléments intangibles (attitudes des vendeurs, atmosphère de l'accueil, odeurs, couleurs,...) dans le succès ou l'échec d'un magasin, au même titre que les éléments plus fonctionnels et plus tangibles (localisation, prix, produits).

Arons (1961) souligne la «complexité de significations et de relations qui caractérise le magasin pour les individus $»^{5}$ tandis que Lindquist (1974) voit en l'image tout ce que l'on peut associer au magasin, qu'il s'agisse des qualités fonctionnelles déjà évoquées par Martineau (assortiment, niveau de prix, facilités de crédit, disposition de la surface de vente) ou d'attributs psychologiques (sentiments d'appartenance, de chaleur et d'amitié, mais aussi d'excitation et d'intérêt). A ces dimensions cognitives et affectives, Coutelle (1998) propose d'ajouter la dimension conative induite par l'expérience d'achat.

Pour Oxenfeldt (1974), la bonne ou la mauvaise image du magasin est fonction de cinq conditions: l'influence du passé sur les images présentes (d'où le décalage entre les impressions et la réalité), l'échec ou le succès d'image des concurrents, les erreurs du magasin et de ses concurrents, le rôle des leaders d'opinions qui louent ou dévalorisent le magasin, enfin les bénéfices consommateurs qui ne sont pas vus ou reconnus par les clients potentiels. Il insiste aussi sur l'interaction entre ses composantes factuelles et ses composantes affectives qui fait de l'image "plus que la somme de ses parties»(p.9). Ce supplément émotionnel permettrait aux clients sans opinion bien établie sur le magasin, y compris à ceux qui ne le fréquentent pas, de toujours avoir un sentiment, une impression à son propos. Rares seraient ainsi les clients qui n'éprouveraient rien face à un magasin ou même qui considèreraient que toute comparaison avec un autre est impossible compte tenu de la diversité des articles présentés, des incessantes variations de prix et des reconfigurations du merchandising.

Cette opinion est contestée, au moins sur un plan méthodologique, par Schiffman et al. (1977) car elle conduit à collecter les perceptions biaisées de personnes connaissant peu le magasin analysé, à côté de celles de clients l'ayant déjà pratiqué, voire de manière encore plus restrictive de clients présents ou achetant dans celui-ci au moment de l'étude (Kunkel et Berry, 1968). Pour Schiffman et al. (1977, p.4), une étude rigoureuse consisterait donc à n'interroger que «les acheteurs connus d'une certaine catégorie de produits, dans un laps de temps donné, pour chaque magasin à comparer», et encore sous contrainte d'un achat d'un montant minimal ${ }^{6}$.

Loin d'être irrationnelle, l'image du magasin combine ainsi à la fois des éléments rationnels et émotionnels (Golden et al., 1987), qui sont perçus et pondérés (Doyle et Fenwick, 1974). Très souvent assimilée à un ensemble d'attitudes (Doyle et Fenwick, 1974) ou à une attitude globale «basée sur les perceptions d'attributs pertinents des magasins » (Steenkamp et Wedel 1991, p.301), elle peut se définir alors comme une «mesure de l'attitude du consommateur à l'égard du point de vente, telle qu'elle résulte de la combinaison de critères d'évaluation du point de vente et de croyances à l'égard du point de vente considérées sur chacun de ces critères $\gg\left(\right.$ Filser, 1985) ${ }^{7}$.

\subsection{Distinction par rapport aux concepts de personnalité et d'atmosphère}

\footnotetext{
${ }^{5}$ Cité par Jain et Etgar (1976, p.61).

${ }^{6}$ Dans leur étude, plus de $100 \$$.

${ }^{7}$ Cité par Jallais et al. (1994).
} 


\subsubsection{Image et personnalité du magasin}

Si l'on se réfère aux définitions du Petit Robert ainsi qu'aux travaux initiaux, le concept d'image intègre à la fois ceux de personnalité («ce qui fait l'individualité d'une personne morale, apparence d'une personne ») et d'atmosphère («le milieu, au regard des impressions qu'il produit sur nous, de l'influence qu'il exerce $»)^{8}$. C'est d'ailleurs ce qui justifie en partie les dimensions affectives de l'image du magasin.

Mais depuis l'emploi du terme de personnalité par Martineau, la délimitation des concepts d'image, de personnalité et d'atmosphère du magasin pose de réels problèmes. Par exemple, les recherches de Baker et al. (2002) ou de Collins-Dodd et Lindley (2003) sont rangées, au gré des revues de littérature, parmi les études d'image ou bien d'atmosphère du point de vente. En effet, elles entremêlent des caractéristiques traditionnellement attachées à l'image telles que la qualité perçue des marchandises ou le personnel de vente et des variables plus atmosphériques comme le décor, l'ambiance ou la musique.

Pourtant, si la mesure de l'image du magasin est « analogue au processus de définition et de mesure de la personnalité » (Hansen et Deutscher 1977, p.63), le concept de personnalité paraît plus restrictif que celui d'image du magasin. La personnalité du magasin est en effet, définie comme «la représentation mentale d'un magasin fondée sur des dimensions qui caractérisent habituellement la personnalité d'un individu» (d'Astous et al. 2002, p.117). Cette définition centrée sur les «traits de caractère » est très proche de celle proposée par Aaker (1997) pour la personnalité de marque. D'Astous et al. (2002) l'évaluent au travers de cinq dimensions : l'enthousiasme (souriant, enthousiaste, plein d'entrain, dynamique, amical, sympathique, audacieux), le raffinement (chic, haut de gamme, élégant, à la mode, snob, riche, sélectif), l'authenticité (honnête, digne de confiance, sincère, vrai, authentique, sûr, consciencieux), la solidité (robuste, solide, reconnu, qui a du succès, leader, imposant, organisé) et le caractère désagréable (agressif, agaçant, criard, superficiel, démodé, rigide).

\subsubsection{Image et atmosphère du magasin}

Le succès d'enseignes telles que Lush ou Abercrombie \& Fitch (Lemoine et Badot, 2009) suffit à montrer à quel point l'atmosphère de vente peut «améliorer l'image du point de vente », même si son impact sur les ventes n'est pas «nécessairement immédiat » (Filser 2004, p.15).

Elle consiste en « la création d'un environnement d'achat produisant chez l'individu des effets émotionnels spécifiques, susceptibles d'augmenter sa probabilité d'achat » (Kotler 1973, p.50). Ceci rejoint l'idée de Donovan et Rossiter (1982) et Donovan et al. (1994) selon laquelle par les facteurs cognitifs qu'elle contient, l'image expliquerait davantage la sélection du magasin et la plupart des achats planifiés, alors que par sa dimension émotionnelle, l'atmosphère déterminerait les achats imprévus effectués dans le magasin. Les variables atmosphériques pourraient en effet, avoir plus d'impact que les autres variables marketing et que le produit lui-même au moment de l'achat (Kotler, 1973 ; Baker et al., 1994 ; Richard, 2005) : en capturant l'attention des clients et en suspendant leur perception du temps (notion de flow de Csikszentmihalyi 1997), elles réduisent le coût psychologique de l'expérience de magasinage (Eroglu et al., 2001 ; Baker et al., 2002) et génèrent des affects (Kotler, 1973) positifs ou négatifs (agoraphobie, pression des vendeurs,...).

\footnotetext{
${ }^{8}$ Petit Robert.
} 
Constituée d'un «amalgame de facteurs tactiles (température, humidité), sonores (musique, bruits), gustatifs (dégustations et produits proposés), olfactifs (odeurs, parfums), visuels (couleurs, lumières, architecture) et sociaux (contacts avec les employés, densité de clients) » (Ettis 2005, p.6) ${ }^{9}$, l'atmosphère affecte les cinq sens, l'odorat semblant, pour des raisons physiologiques, celui le plus attaché à l'émotion (Michon et al., 2005).

Néanmoins, les indications fournies par le design du magasin semblent jouer le premier rôle :

1) en raison de la supériorité de l'image visuelle sur le message écrit ou musical (Dual encoding model de Paivio 1991), elles influencent davantage le choix des clients, que ne le feraient les employés du magasin et la musique diffusée (Baker et al., 2002) ;

2) elles accroissent le sentiment de contrôle personnel, donc de plaisir, mais aussi la perception de qualité des produits vendus (Hui et Bateson, 1991);

3) elles constituent un élément prédictif du prix perçu (beau magasin = produit cher, magasin sommaire $=$ prix discount) ;

4) elles délivrent des indications implicites de normes et de comportements attendus sur le lieu de vente (« signes, symboles et artefacts » de Bitner).

Se référant aux travaux de Broadbent et al. (1980) et de Rappoport (1982), Bitner (1992, p.62) souligne ainsi que «l'environnement peut être vu comme une forme non verbale de communication », notamment dans le cas des services et des magasins en libre-service où les indications extrinsèques permettent aux clients d'inférer sur la qualité (Zeithaml, 1988). De plus, en raison de l'interdépendance de chaque stimuli, l'environnement et plus généralement l'atmosphère sont perçus par les individus de façon holistique (Bitner, 1992); d'où leur difficulté à verbaliser et à mémoriser qui peut expliquer celle des chercheurs à démontrer ses effets (Donovan et Rossiter, 1982). Ceux-ci sont le plus souvent évalués à partir de la typologie PAD (Russel et Mehrabian, 1976; Baker et al., 2002), le plaisir et l'activation (sommeil ou surexcitation) semblant mieux expliquer, selon Donovan et Rossiter (1982), les intentions comportementales en magasin que la dominance (sensation de pouvoir et de contrôle). Reprenant les conclusions de Kaplan (1987), Bitner (1992, p.63) estime quant à elle, qu'un «environnement particulier peut être prédit par trois dimensions : la complexité (...), le mystère et la cohérence».

En définitive, quoique la personnalité et l'atmosphère soient historiquement intégrées à l'image du magasin, la multiplication des recherches axées sur l'un ou l'autre de ces concepts devrait à terme limiter par défaut l'image à des «attributs concrets et relatifs à un bénéficeconsommateur» (Ladwein 1998, p.17). D'ailleurs, le fait que l'atmosphère de vente soit principalement liée à l'exploration d'un lieu physique ou virtuel dans un contexte donné, la relie désormais davantage au concept d'expérience qu'à celui d'image ; en témoignent les travaux sur le réenchantement des espaces commerciaux (Hetzel, 2000 ; Andrieu et al., 2004 ; Badot, 2005) ou dans le domaine du commerce électronique, celles reliant webmosphere et expérience de flow (Gharbi et al., 2002).

\subsection{Distinction par rapport aux concepts de qualité et d'expérience de magasinage}

Même s'ils sont plus récents que les concepts de personnalité ou d'atmosphère, ceux de qualité du magasin et d'expérience de magasinage sont également connexes à celui d'image.

\subsubsection{Image et qualité du magasin}

\footnotetext{
${ }^{9}$ Pour une synthèse des composantes de l'atmosphère du point de vente, voir Turley et Milliman (2000).
} 
Initialement définie comme "la mesure dans laquelle un produit ou service rencontre ou dépasse les attentes » (Reeves et Bednar 1994, p.432), la qualité a connu au fil du temps des conceptualisations très variables (excellence, valeur, conformité à un cahier des charges ou aux besoins,...). Chevauchant souvent le concept de satisfaction, soit comme antécédent, soit comme conséquence, elle paraît difficile à transposer dans le secteur de la distribution. Gaur et Agrawal (2006) relèvent ainsi que la plupart des recherches (Carman, 1990 ; Finn et Lamb, 1991) ont échoué à appliquer les mesures de qualité SERVQUAL ou RSQS à ce secteur. Mehta et al. (2000) $)^{10}$ ont également montré que cette dernière échelle était meilleure pour certains distributeurs (supermarchés) que pour d'autres (détaillants de produits électroniques). En effet, «l'expérience dans un magasin de détail diffère significativement » de celle dans un autre secteur «par la façon dont les clients négocient leur parcours, trouvent les marchandises qu'ils veulent, interagissent avec le personnel en cours de parcours, retournent la marchandise, toutes choses qui influent sur la qualité perçue du service » (Gaur et Agrawal 2006, p.319).

Dans le domaine du commerce électronique, ce concept est encore souvent préféré à celui d'image (Loiacono et al., 2007 ; Tarafdar et Zhang, 2007 ; Bressolles et Durieu, 2008) parce qu'il a l'avantage de se concentrer sur les caractéristiques du canal qui peuvent le plus empêcher le dénouement d'une transaction. Il paraît cependant de moins en moins pertinent pour comprendre les «sources de valeur» (Aurier et al., 2000, p.2). En effet, il reste encore trop tourné vers l'interne (Aurier et al., 2004) sans prendre en compte des critères marketing plus spécifiques à l'enseigne. Ceci a d'ailleurs conduit Rolland et Freeman (2010) à intégrer dans leurs travaux sur la qualité du site, des dimensions propres à l'image telles que l'offre ou le niveau de prix. Cette critique s'applique tout autant aux points de vente physiques, dans lesquels les visites mystères contrôlent bien mieux la qualité de service escomptée par le détaillant qu'elles ne permettent d'évaluer et de recadrer l'image de ses magasins.

\subsubsection{Image et expérience de magasinage}

Bien que se nourrissant de «la totalité des expériences »(Berry 1969, p.5), «sensations et pensées » accumulées au fil du temps par le client ou le visiteur (Ingarao 2004, p.51), l'image du magasin semble se distinguer de l'expérience de magasinage sur plusieurs points :

1) tout individu peut avoir l'image d'un point de vente même sans l'avoir fréquenté (Oxenfeldt, 1974 ; Kerin et al., 1992) alors que l'expérience, «définie comme une interaction entre une personne et un objet de consommation, dans une situation donnée (paradigme P.O.S «personne x objet x situation» de Punj et Stewart $1983^{11}$ ) », doit nécessairement être vécue ;

2) l'expérience met davantage l'accent sur les «émotions et les sens vécus pendant l'immersion au détriment de la dimension cognitive » (Carù et Cova 2003, p.273) ;

3) alors que par son immédiateté (Bouchet, 2004), l'expérience « possède un ancrage temporel » précis (Roederer 2008, p.6), l'image fait davantage appel à une impression générale mémorisée. L'expérience de magasinage constitue donc une « interaction sociale particulière au sein d'un espace marchand » (Bouchet 2004, p.63).

A cela, Kerin et al. (1992, p.380) ajoutent que l'expérience de magasinage ne mobilise «que les attributs intrinsèques du magasin, excluant le prix des produits et les considérations de qualité qui affectent les perceptions » des clients. Mais cet argument paraît moins recevable puisque la valeur d'expérience appliquée à la vente à distance (catalogue et Internet) par Mathwick et al. (2001) intègre par exemple, dans sa dimension économique, le rapport qualité/prix.

\footnotetext{
${ }^{10}$ Cités par Gaur et Agrawal (2006).

${ }^{11}$ Cités par Roederer (2008).
} 
En synthèse, l'image du magasin semble désormais faire appel à des éléments essentiellement cognitifs, tandis que la personnalité et l'atmosphère se réfèrent davantage à des éléments affectifs et l'expérience de magasinage à des éléments conatifs. Etant moins focalisée sur le processus transactionnel, elle est aussi moins restrictive que le concept de qualité (lui aussi à dominante cognitive). Une modélisation empirique pourrait valider et approfondir ce travail conceptuel, en positionnant l'image du magasin dans le processus de décision des consommateurs. Il s'agirait notamment de montrer que la personnalité, l'atmosphère ou la qualité ne sont pas des substituts, mais des antécédents de l'image, alors que l'expérience en est une conséquence.

Une fois définie et délimitée l'image du magasin, examinons à présent ses effets sur les comportements des consommateurs.

\section{L'image du magasin : ses effets sur les comportements}

Si de multiples recherches ont été menées sur l'image du magasin et sa mesure, peu d'entre elles ont examiné ses effets sur les comportements des consommateurs et la performance des distributeurs. Sa contribution au processus de décision du consommateur fait pourtant peu de doute (Nevin et Houston, 1980 ; Joyce et Lambert, 1996 ; Grewal et al., 1998). L'image du magasin est en effet, « un prédicteur du choix du détaillant (Grewal et al., 1998 ; Hildebrandt, 1988 ; Schiffman et al., 1977), un construit clé dans la compréhension des inférences sur la qualité du magasin et du produit (Baker et al., 1994 ; Darden et Babin, 1994), un prédicteur de la satisfaction (Bloemer et de Ruyter, 1998), un antécédent de la position concurrentielle (Burt et Carralero-Encinas, 2000 ; Pessemier, 1980), et un prédicteur de la fidélité au magasin (Bellenger et al., 1976 ; Lessig, 1973 ; Sirgy, 1985)» (Hartman et Spiro 2005, p.1112). La figure 1 suggère d'ailleurs que les caractéristiques d'image du magasin comptent parmi les quatre types de variables qui influencent le choix d'un point de vente dans un «système multi-localisé » (Nicholson et Vanheems 1998, p.109).

Figure 1 - Modèle de choix d'un point de vente d'Engel, Blackwell et Miniard

(Nicholson et Vanheems 1998, p.109)

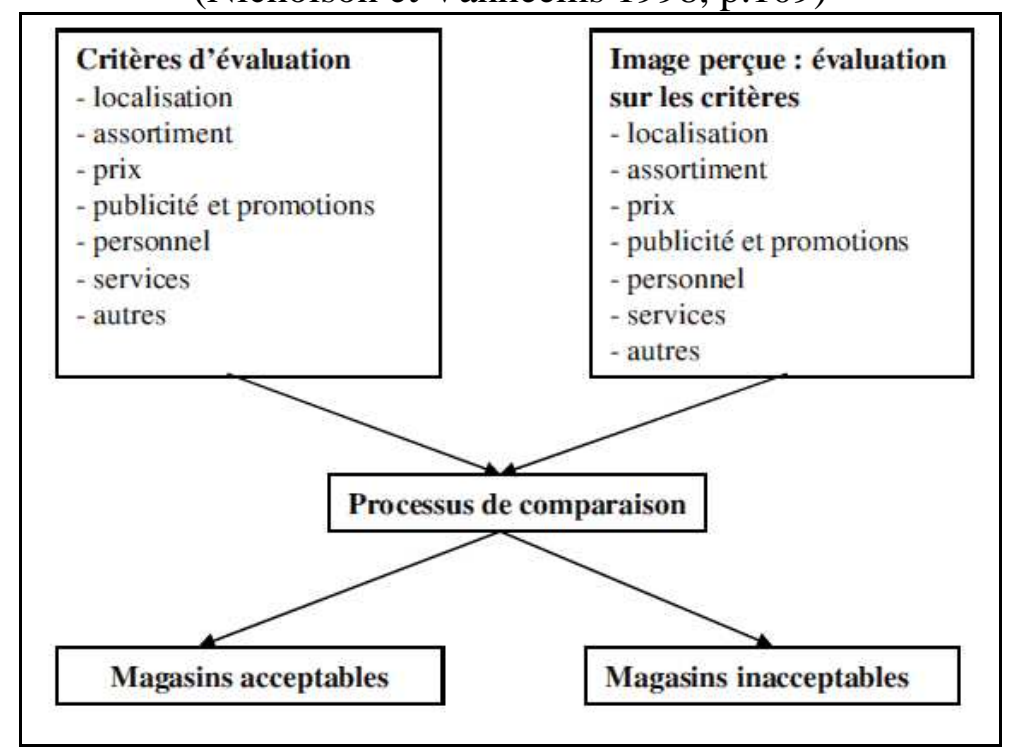

Une image différenciatrice du magasin constitue donc l'une des principales sources de positionnement pour le commerce de détail (Filser, 2004) et une condition pour que 
l'enseigne maintienne un avantage concurrentiel non duplicable sur le marché (Steenkamp et Wedel, 1991).

\subsection{L'image du magasin, source d'attraction}

Dans les modèles gravitaires ou de localisation-allocation, la proximité et la masse constituent les critères majeurs pour expliquer et prédire les comportements de fréquentation d'un magasin : selon une étude empirique citée par Bell (1999), la distance expliquerait $70 \%$ du choix d'un supermarché.

Mais de l'aveu même de Huff ${ }^{12}$, une insuffisante prise en compte de l'image des magasins est susceptible de limiter l'efficacité de son modèle car une image favorable peut élargir le périmètre d'attraction d'un point de vente, compenser une localisation malcommode (Stanley et Sewall, 1976), motiver les clients pour qu'ils achètent là plutôt qu'ailleurs, les faire «aller presque automatiquement à ce magasin pour l'article dont ils ont besoin » (Oxenfeldt 1974, p.9), voire les faire venir lorsqu'ils «n'ont rien à faire de mieux » (Oxenfeldt 1974, p.9). C'est pourquoi Jallais et al. (1994, p.157) estiment que l'ensemble des caractéristiques du magasin pèseraient « entre 15 et $20 \%$ dans la décision de fréquentation d'un magasin ». Avec l'urbanisation et la mobilité croissante de la société, la commodité et notamment sa composante «distance» pourrait en outre, selon Doyle et Fenwick (1974), perdre de l'importance au profit des variables d'image (prix, assortiment, réputation, disposition du magasin,...).

Bucklin (1966), Gautschi (1981), Stanley et Sewall (1976) ou Osman (1993) militent donc pour la prise en compte de critères plus subjectifs, contenus dans l'image. Bucklin montre par exemple que la communication, le prix et la commodité ne sont pas insignifiants par rapport à la distance et à la masse. Dans une recherche de Gentry et Burns (1977) sur les motifs de fréquentation des centres commerciaux, la proximité par rapport au domicile ne constituerait que le $14^{\text {ème }}$ critère de décision de fréquentation des centres commerciaux, derrière les variables d'image (capacités de parking, horaires d'ouverture, type de clients, rapport qualitéprix, publicité, variété des produits, réputation des magasins, propreté, qualité et variété des magasins, aménagement du complexe), mais redeviendrait le critère déterminant lorsque les chalands évaluent trois centres commerciaux, non plus en absolu mais en relatif.

Remarquons néanmoins que la proximité perçue est en partie prise en compte dans l'image du magasin, au travers de critères subjectifs tels que la facilité d'accès ou la praticité ; Chardon et Dumartin (1998) évaluent ainsi en moyenne à $67 \%$, l'impact de la praticité (proximité, facilité d'accès) sur le choix d'une grande surface alimentaire.

Par ailleurs, il est nécessaire de distinguer l'impact de l'image sur la fréquentation du point de vente et celui sur les comportements d'achats. En effet, l'image influence différemment le choix d'un magasin ou bien le panier moyen (N'Gobo, 2006). Dans le cas des centres commerciaux (Nevin et Houston, 1980) ou des magasins économiques (Kelly et Smith, 1983), le modèle qui combine distance et image améliore peu ou pas les prédictions, sauf pour les clients les moins réguliers. Par contre, Kelly et Smith (1983) montrent que la qualité des produits, la courtoisie des employés et le niveau de prix sont déterminants pour prédire les comportements d'achat. Il paraît toutefois difficile de hiérarchiser une fois pour toutes, comme le font Pan et Zinkhan (2006), les dimensions qui influencent le plus le choix du magasin et sa fréquentation, tant leur impact dépend des avantages recherchés pour chaque type de magasin considéré.

\footnotetext{
${ }^{12}$ Cité par Nevin et Houston (1980).
} 
A l'heure du commerce électronique et des drive in, il serait donc intéressant de renouveler les études combinant image et proximité afin d'identifier les conditions situationnelles dans lesquelles l'image du point de vente joue un rôle majeur, pour quels types d'enseignes et de formats de magasins.

\subsection{Ses effets sur les intentions d'achat et de fidélité}

Longtemps la théorie a fait l'hypothèse qu'il était «indifférent pour un consommateur d'acheter un produit dans le magasin $\mathrm{A}$ ou le magasin $\mathrm{B}$ à partir du moment où il trouvait dans l'un ou l'autre point de vente le même produit de la même marque au même prix et avec un niveau de service équivalent »(Jallais et al. 1994, p.255). Or, au-delà de ses missions commerciales classiques de mise à disposition des produits ou des services, de génération de trafic et d'accroissement du panier moyen, le magasin est un média, qui «à travers ses composantes concourt à l'image que se forge le consommateur» (Jallais et al. 1994, p.255).

Pourtant, si de nombreuses recherches ont exploré les caractéristiques saillantes de l'image des points de vente, peu d'entre elles ont cherché à les «relier au choix du magasin et à développer des modèles prédictifs des dépenses » qui y sont réalisées (Malhotra 1983, p.3). Selon le type de magasins et les caractéristiques personnelles des individus, Malhotra souligne une relation entre certaines dimensions saillantes et la préférence pour tel ou tel magasin. De son côté, Pessemier (1980, p.100) estime que la préférence pour un magasin est d'autant plus forte que son image est proche de «l'idéal ou du meilleur niveau possible ».

Le choix du lieu d'achat précédant généralement celui du produit ou de la marque (Bell, 1999), son image a également une influence sur la qualité perçue des marques vendues (Semeijn et al., 2004), sur la confiance du consommateur (Zimmer et Golden, 1988 ; Gurviez et Korchia, 2002) et sur les intentions d'achat (Grewal et al., 1998).

En règle générale, une image favorable est aussi supposée conditionner la fidélité au magasin (Mazursky et Jacoby, 1986 ; Osman, 1993), au moins tant que les attributs jugés importants par le client sont favorablement évalués ou qu'ils peuvent être compensés par des attributs alternatifs (Osman, 1993). Pour fidéliser les clients qui butinent entre différents points de vente pour le même type de produits, les managers d'enseigne devraient donc affirmer une image spécifique (Schiffman et al., 1977). Sans quoi, avec une "personnalité émoussée » (Martineau 1958, p.50), leur magasin ne constituera jamais qu'une alternative par rapport au lieu d'achat préféré par les clients.

La liaison entre image et fidélité au magasin pose néanmoins deux questions. D'abord, est-ce l'image favorable du magasin qui attire, ou bien l'image défavorable des magasins concurrents qui repousse ? Lessig (1973) opte pour une fidélité dominée par l'évitement des autres magasins plutôt que par l'attraction pour un magasin précis ; ceci devrait selon lui, inciter les managers à s'intéresser davantage à l'image des magasins concurrents qu'à celle de leur propre lieu de vente. Ensuite, cette relation est-elle directe ou bien passe-t-elle par la satisfaction? Bloemer et De Ruyter (1998) testent une relation indirecte entre l'image du magasin et la fidélité, via la satisfaction (Figure 2).

Figure 2 - Modèle empirique de Bloemer et De Ruyter (1998, p.509) 


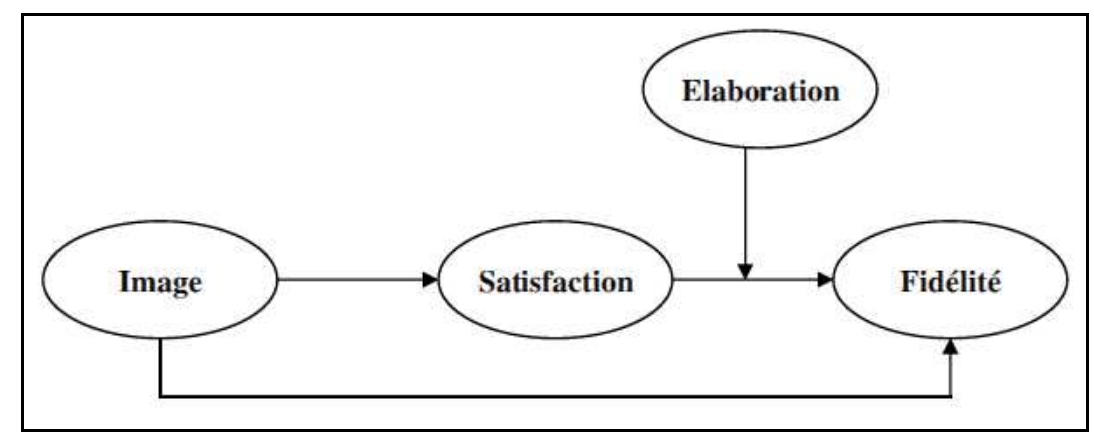

Pour cela, ces auteurs distinguent la satisfaction latente (faible élaboration) de la satisfaction manifeste (haut niveau d'élaboration), c'est-à-dire la simple acceptation du magasin et l'engagement. Ils aboutissent à la conclusion qu'il n'existe pas de lien direct entre l'image du magasin et la fidélité. En effet, selon eux, «la satisfaction vis-à-vis du magasin transforme les conséquences de l'image du magasin » (Bloemer et De Ruyter 1998, p.510), la satisfaction manifeste renforçant davantage la fidélité que la satisfaction latente. Ceci tendrait à remettre en cause l'intuition de certains auteurs, notamment d'Hirshman sur un lien direct entre image et fidélité. Cependant, un terrain limité (300 questionnaires) et spécifique (un grand magasin de la Suisse allemande) ainsi qu'un construit d'image du magasin bâti à partir de seulement huit items issus qui plus est, d'un manuel ${ }^{13}$ incitent pour le moins à la prudence.

Finalement, il semblerait que la relation entre l'image du magasin et la fidélité soit à la fois directe et indirecte via la satisfaction : cette double relation est confirmée par Hsu et al. (2010) pour lesquels l'influence de l'image sur les intentions comportementales est essentiellement indirecte, mais aussi par Helgesen et al. (2010) qui concluent à une influence plus directe qu'indirecte.

De manière plus générale, l'accent mis par Bloemer et De Ruyter sur le degré d'implication des clients incite à s'interroger sur les caractéristiques personnelles (genre, âge, orientations d'achat,...) susceptibles de modérer la perception des magasins tant au niveau des dimensions d'image privilégiées, qu'à celui du mode de traitement mis en œuvre (holistique ou analytique).

Une ultime question se pose alors : comment mesurer l'image du magasin et comment identifier les éléments qui la sous-tendent?

\section{L'image du magasin : sa mesure}

La mesure de l'image du magasin, tout comme son périmètre et ses effets, donne lieu à de nombreux débats. Ceux-ci portent principalement sur les dimensions qui sous-tendent la perception par les consommateurs de l'image du magasin et sur la manière dont l'image est perçue (Zimmer et Golden, 1988).

\subsection{Les dimensions sous-tendant l'image du magasin}

Les successeurs de Martineau ont multiplié les études de cas destinées à identifier les caractéristiques d'image du magasin (Tableau 1). Or, cette prolifération pose problème : «le nombre des composants est incertain et paraît dépendre des méthodes de catégorisation des chercheurs » (Hirschman et al. 1978, p.3) d'autant que peu de ces études ont été régulièrement dupliquées pour «d'autres sites ou d'autres temps » (Hirschman et al 1978, p.4).

\footnotetext{
${ }^{13}$ Celui de Ghosh (1990).
} 
Tableau 1 - Synthèse des caractéristiques d'image identifiées par les principaux auteurs

\begin{tabular}{|c|c|}
\hline Auteurs & Critères \\
\hline Martineau (1958) & $\begin{array}{l}\text { Disposition et architecture des locaux } \\
\text { Symboles et couleurs } \\
\text { Communication } \\
\text { Personnel de vente (courtoisie, adéquation) }\end{array}$ \\
\hline Kunkel et Berry (1968) & $\begin{array}{l}\text { Style, médias utilisés, fréquence } \\
\text { Attitudes, compétence, nombre, qualité du service } \\
\text { Prix des produits } \\
\text { Marques } \\
\text { Largeur, profondeur de l'assortiment } \\
\text { Nouveauté des produits } \\
\text { Proximité maison, travail, accès } \\
\text { Parking, plages d'ouverture, commodité } \\
\text { Crédit, livraison, restauration, garderie,... } \\
\text { Mise en scène ou évènement promotionnel, bonus } \\
\text { Disposition des locaux, décor intérieur et extérieur, disposition des produits, } \\
\text { types de clients, encombrement, prestige } \\
\text { Reprises, échanges, loyauté }\end{array}$ \\
\hline Oxenfeldt (1974) & $\begin{array}{l}\text { Facteurs tangibles et réels : qualité (best value), largeur et profondeur de } \\
\text { l'assortiment, ruptures de stocks minimisées, style, rapidité / personnalisation, } \\
\text { crédit, assistance à la vente, vitesse de paiement, facilités de retour et de } \\
\text { réparation } \\
\text { Bénéfices intangibles mais authentiques : ambiance relationnelle amicale, plaisir, } \\
\text { familiarité, confiance, entraide, gratification et reconnaissance personnelle, } \\
\text { propreté } \\
\text { «Fantasies »: «the best people shop there; known to be the best store »; you'll } \\
\text { meet fascinating people there ... ») }\end{array}$ \\
\hline Lindquist (1974) & $\begin{array}{l}\text { Escalators, lumière, air conditionné, toilettes, largeur des allées,... } \\
\text { Parking, commodité d'accès } \\
\text { Vente, présence du self-service, retour des produits, livraison, facilité de crédit } \\
\text { Publicité, présentation, symboles et couleurs } \\
\text { Qualité, assortiment, style, garantie, prix } \\
\text { Statut social du magasin, congruence avec l'image de soi, personnel } \\
\text { Ambiance de chaleur et d'appartenance } \\
\text { Conservateur ou moderne, réputation, sûreté } \\
\text { Retour des produits, satisfaction en général }\end{array}$ \\
\hline $\begin{array}{l}\text { Hansen et Deutscher } \\
\text { (1977) }\end{array}$ & $\begin{array}{l}\text { Produit (qualité, sélection, style, prix) } \\
\text { Service (possibilité de mettre des articles de côté, personnel de vente, facilité de } \\
\text { retour, crédit, livraison,) } \\
\text { Clientèle } \\
\text { Facilités physiques, propreté, disposition du magasin } \\
\text { Commodités (localisation du magasin, facilités de shopping, attractivité, parking) } \\
\text { Promotion / publicité } \\
\text { Atmosphère (sympathie congruence) } \\
\text { Institutionnel : réputation du magasin } \\
\text { Satisfaction après vente }\end{array}$ \\
\hline Schiffman et al. (1977) & $\begin{array}{l}\text { Commodité de lieu } \\
\text { Prix compétitifs ou bons deals } \\
\text { Politiques de garantie } \\
\text { Service en magasin } \\
\text { Expertise des vendeurs } \\
\text { Choix produit }\end{array}$ \\
\hline Pessemier (1980) & $\begin{array}{l}\text { Profil de la clientèle } \\
\text { Cycle de vie du magasin } \\
\text { Offres produits (y compris prix et marques) }\end{array}$ \\
\hline
\end{tabular}




\begin{tabular}{|c|c|}
\hline & $\begin{array}{l}\text { Commodité de lieu } \\
\text { Plaisir du shopping } \\
\text { Facilité de transaction } \\
\text { Promotion } \\
\text { Probité } \\
\text { Force et clarté d'image }\end{array}$ \\
\hline Mazursky et Jacoby (1986) & $\begin{array}{l}\text { Nombre de vendeurs et de caissiers par département } \\
\text { Politique de retour des produits } \\
\text { Nombre de salons d'essayage } \\
\text { Acceptation de la carte de crédit } \\
\text { Localisation } \\
\text { Nombre d'étages } \\
\text { Design intérieur du magasin } \\
\text { Marques des produits } \\
\text { Niveau de prix } \\
\text { Assortiment } \\
\text { Pourcentage de stock en vente } \\
\text { Texture de la marchandise } \\
\text { Remise sur ventes } \\
\text { Couleurs des produits }\end{array}$ \\
\hline Zimmer et Golden (1988) & $\begin{array}{l}\text { Bonne disposition et apparence des locaux } \\
\text { Mauvaises conditions physiques du magasin } \\
\text { Qualité de la marchandise } \\
\text { Commentaires sur la publicité } \\
\text { Commentaires positifs sur la vente } \\
\text { Commentaires négatifs sur les techniques mises en œuvre durant la vente } \\
\text { Sélection } \\
\text { Service } \\
\text { Commentaires sur le crédit } \\
\text { Commentaires positifs sur le catalogue } \\
\text { Mauvais service catalogue } \\
\text { Commentaires sur la garantie et les retours } \\
\text { Bonne impression sur le personnel de vente } \\
\text { Localisation } \\
\text { Prix } \\
\text { Bonne réputation } \\
\text { Magasin vulgaire (low-class) } \\
\text { Magasin en désordre } \\
\text { Commentaires sur la catégorie de magasin } \\
\text { Similaire aux autres magasins } \\
\text { Intéressant pour des produits spécifiques } \\
\text { Bonne réparation } \\
\text { Autres }\end{array}$ \\
\hline
\end{tabular}

Pour rendre exploitable une telle diversité d'attributs d'image sans en perdre la substantifique moelle, les chercheurs ont émis trois types de propositions.

La première proposition vise à classer ces critères sans renoncer à aucun. Hansen et Deutscher (1977) proposent ainsi de distinguer trois niveaux de catégorisation: la dimension (par exemple, la marchandise), le composant (par exemple, la sélection de cette marchandise), l'attribut (par exemple, une question relative à la sélection de cette marchandise). De son côté, Oxenfeldt (1974) répartit les dimensions d'image en trois ordres de natures très différentes : les facteurs tangibles et réels, les bénéfices intangibles mais authentiques, et enfin les fantasies.

La seconde proposition ne prend en compte que les critères auxquels les consommateurs attachent de la valeur. Myers et Alpert (1977) proposent de ne retenir que les attributs à la fois importants (motif réel de fréquentation) et distinctifs (vraie différence avec les magasins 
concurrents) ; Dubois (1980) y ajoute le critère de saillance (présence de l'attribut dans l'esprit du consommateur au moment où celui-ci passe à l'acte). C'est aussi l'option retenue par Kunkel et Berry (1968, p.25) pour ne pas «forcer les réponses pour toutes les composantes de l'image », même celles qui n'évoquent rien à la personne interrogée; ils préconisent le recours à des questions totalement ouvertes plutôt qu'à des tests de différences sémantiques.

Il en va de même pour James et al. (1976) qui utilisent le modèle multi-attributs d'analyse des attitudes et des croyances élaboré par Rosenberg et Fishbein, pour dégager uniquement 5 à 9 croyances saillantes, plutôt que les nombreux critères sémantiques différentiels fréquemment utilisés. Dans le cas du magasin de prêt-à-porter masculin qu'ils étudient, 6 attributs ressortent : l'assortiment, le personnel, l'atmosphère, le service, la qualité et le prix. Ils en concluent à la supériorité descriptive et prédictive de ce modèle multi-attribut par rapport aux échelles sémantiques différentielles utilisées antérieurement tant pour sélectionner les attributs saillants que pour les pondérer.

Dans ce même ordre d'idée, Pessemier (1980) fait le choix astucieux d'évaluer la clarté et la force de l'image (considérées par lui comme un attribut) à partir de la proportion observée de répondants « ne sais pas ».

La troisième proposition fait figure de synthèse des deux premières. Après avoir identifié 15 critères qui influencent la perception de qualité de service, de qualité du produit et de plaisir de la visite, Mazursky et Jacoby (1986) hiérarchisent quatre degrés d'évaluation du magasin : l'information sur les prix (niveau de prix, soldes et promotions), l'offre produit (assortiment, couleurs, marques), la composante physique du magasin (implantation et aménagement) et la politique d'information (nombre de vendeurs par rayon et politique de reprise des produits). Ces auteurs recourent à une représentation en cercles concentriques, avec des degrés de perception interdépendants s'inspirant de Coxon (1982), qui donnent la prépondérance aux attributs centraux sur les attributs périphériques. Pour ces auteurs, si une variation de l'image prix peut grandement modifier la quasi-totalité des autres attributs de l'image du magasin, à l'inverse la modification d'un attribut périphérique n'aurait que peu d'effet sur les attributs centraux.

Mais ce dernier travail sur les éléments centraux et périphériques de l'image du magasin mériterait d'être réitéré sur différents types de commerces, et en se référant plus fidèlement aux modèles ELM ou HSM de persuasion. Il s'agirait en particulier de réévaluer l'impact de l'image-prix sur l'image des magasins et les conditions dans lesquelles un effet-prix peut intervenir.

En effet, Mazursky et Jacoby (1986) ou Morschett et al. (2005), comme les distributeurs interrogés par Oppewal et Timmermans (1997), considèrent le prix comme une dimension centrale. En revanche, la majorité des chercheurs estiment qu'il constitue une dimension périphérique du système de persuasion puisqu'il est extrinsèque au produit, aisément manipulable et agit comme un signal chez le consommateur novice ou désimpliqué (Purohit et Srivastava, 2001).

Une telle proposition pourrait être validée en démontrant que le prix guide davantage le jugement holistique émis par des sujets désimpliqués dans l'acte d'achat, alors qu'une évaluation plus analytique menée par des sujets plus impliqués relativise l'incidence du prix par rapport à d'autres dimensions constitutives de l'image du magasin. Ce pont fait entre les évaluations holistiques et analytiques de l'image du magasin ouvre certainement une piste de recherche prometteuse tant en termes théoriques qu'opérationnels.

\subsection{La manière dont l'image du magasin est perçue}


Oxenfeldt (1974) ainsi que Zimmer et Golden (1988) sont certainement les auteurs qui se sont le plus élevés contre l'emploi de grilles d'attributs susceptibles de décrire a priori la totalité du magasin. En effet, pour eux, les recherches par attributs «ne peuvent capturer qu'une partie de l'image que le client a du commerçant » (Zimmer et Golden 1988, p.265) : une image aux dimensions distinctes, mais où les outputs s'avèrent être plus que la simple somme des inputs. Zimmer et Golden estiment ainsi qu'un tiers de la perception de l'image finale aurait été amputée de leur étude, si elles s'en étaient tenues aux seuls attributs spécifiques. En effet, ceux-ci tendent à sous-estimer la dimension affective (Cohen et Basu, 1987). Zimmer et Golden concluent que les échelles sémantiques différentielles, les échelles multidimensionnelles ainsi que les échelles non métriques «ne parviennent généralement pas à capturer la gelstat de l'image du magasin » (p.266).

S'élevant contre les biais liés aux présupposés des chercheurs dans le choix de leurs critères, ces auteurs recourent donc à des construits non structurés, c'est-à-dire aux « descriptions d'image spontanées de la part des consommateurs, sans diriger les répondants vers des dimensions affectives ou des attributs spécifiques »(p.268). Pour déterminer la nature de la perception des consommateurs, elles utilisent l'analyse de contenu développée par Berelson (1954) qui requiert des règles explicitement formulées d'inclusion ou d'exclusion des catégories. La méthodologie scrupuleuse qu'elles utilisent, leur permet à partir de questions totalement ouvertes d'obtenir 220 termes qualifiant l'image de trois magasins, qui se synthétisent en 47 attributs spécifiques ainsi qu'en des images évoquées qui transcendent les perceptions de ces attributs spécifiques. Zimmer et Golden soulignent tout de même, les limites de l'analyse de contenu : difficulté à coder et à quantifier l'information (vs échelles de mesure), charge de travail des répondants (écrit totalement libre), surpondération de certains répondants plus diserts que d'autres, inégalité dans la concision et la précision de pensée des répondants.

Cependant, même si la façon de traiter l'information tend à transformer les indications visuelles mobilisées dans le processus d'inférence, Chowdhury et al. (1998) concluent que les deux méthodes d'évaluation de la perception (construits structurés pour Hirschman et al. ; construits non structurés pour Zimmer et Golden) aboutissent à des résultats très proches, avec un avantage aux échelles structurées pour expliquer la variance.

Les dimensions qui peuvent caractériser l'image du magasin sont d'ailleurs globalement convergentes. Selon les auteurs et leur degré de détail dans les attributs, Jara (2006) comptabilise entre 5 et 19 critères. En général, l'offre produits domine la perception (Lindquist, 1974), suivie par l'accessibilité du magasin, sa réputation, son service à la clientèle, son ambiance et enfin ses promotions (Thang et Tan, 2003) ${ }^{14}$. Cette hiérarchie peut cependant varier selon l'aire géographique (Hirschman et al., 1981) ou le type de commerces considérés (Arnold et al., 1978).

\section{Conclusion}

L'image du magasin influe sur sa fréquentation ainsi que sur les intentions d'achat et de fidélité ; selon les cas, elle intensifie voire compense les effets de la proximité. Par sa capacité à prendre en compte les caractéristiques essentielles des canaux de distribution à partir des différentes variables du retailing mix (Bloemer et De Ruyter, 1998), elle permet de positionner le point de vente en le plaçant «dans la tête du consommateur sur une carte

\footnotetext{
${ }^{14}$ Notons que ces auteurs n'ont pas pris en considération le prix.
} 
perceptive » (Louis et Pederzoli 2005, p.3) et en le comparant aux magasins concurrents (Hirschman et al., 1981).

Comme toute image, elle constitue une représentation subjective et réductrice. Composée d'éléments tangibles et intangibles, rationnels et émotionnels, elle s'articule autour d'une communauté de perceptions, plus ou moins large et plus ou moins partagée par les différentes parties prenantes (clients, personnel,...).

Néanmoins, malgré une solidité et une antériorité indiscutables, les fondements de l'image du magasin sont de plus en plus remis en cause par l'autonomisation de concepts plus affectifs (atmosphère, personnalité) ou plus cognitifs (qualité) qui y étaient initialement intégrés. Ils sont aussi concurrencés par de nouvelles approches centrées sur l'expérience de magasinage.

Au-delà des pistes théoriques qui ponctuent cet état de l'art, la principale implication managériale concerne le choix du concept auquel les détaillants doivent recourir pour analyser leurs magasins. L'étude d'image semble particulièrement indiquée pour expliquer la sélection de son magasin par rapport à ses concurrents physiques ou même virtuels, dans le cadre d'achats planifiés, à partir de critères essentiellement cognitifs donc aisément activables par l'enseigne (choix, prix, services, promotions,...). La technique utilisée est toujours déclarative (orale ou écrite, directive ou totalement ouverte). Il n'est pas nécessaire que les personnes interrogées soient présentes sur le lieu de vente lors de l'enquête puisque l'image consiste en une sédimentation d'expériences et de perceptions saillantes ${ }^{15}$, ni qu'elles aient une excellente connaissance du magasin, notamment si l'on cherche à comprendre comment attirer de nouveaux clients.

A l'inverse, l'atmosphère ou l'expérience paraissent plus pertinentes pour expliquer des comportements d'achat à l'intérieur du lieu de vente, en particulier le déclenchement d'achats impulsifs. Les techniques utilisées sont alors davantage basées sur l'analyse du non-dit, au moyen de méthodes d'observations (participantes ou sémiotiques), ou d'analyses ethnographiques nécessitant une forte interaction entre la situation et l'objet étudiés et donc s'appliquant peu à des prospects absolus ou relatifs.

Les frontières de l'image du magasin sont surtout menacées par l'extension souvent injustifiée de l'image de l'enseigne ou plus encore, du capital magasin qui, pour Hartman et Spiro (2005), intègre à la fois la performance du point de vente et les comportements des consommateurs par analogie avec le capital-marque (Keller, 1993).

L'enseigne constitue en effet, un concept multiformes, qui fédère de multiples entités : plusieurs canaux de distribution (site et points de vente), parfois différents formats de magasins déclinant le nom du distributeur (exemple : Carrefour), fréquemment une offre de produits MDD,... Réputée plus stable car moins spécifique que celle des magasins (HuvéNabec, 1998), l'image du détaillant peut donc être définie comme «tout ce qui est lié à l'enseigne, de près comme de loin, dans la mémoire du consommateur » (Huvé-Nabec 2002, p.603). Elle peut alors intégrer un grand nombre de caractéristiques tangibles ou intangibles des canaux (personnalité, atmosphère), des bénéfices fonctionnels, expérientiels et symboliques, voire les attitudes globales associées à l'enseigne (Keller, 1993).

Par là même, la mesure de l'image de l'enseigne pose un réel problème de définition conceptuelle et psychométrique. Faut-il la mesurer à partir d'indicateurs principalement issus du retailing mix et servant initialement à évaluer l'image des magasins ? Comment alors pallier le risque de ne plus pouvoir discriminer la manifestation «enseigne » et la manifestation «magasins », comme c'est le cas d'Ailawadi et Keller (2004), de Louis et al. (2006) ou encore de Wang et al. (2009) ? Ne vaut-il pas mieux alors transposer les travaux sur

\footnotetext{
${ }^{15}$ Ceci autorise l'usage d'un questionnaire en ligne, sous réserve des biais liés à l'échantillonnage.
} 
l'image de marque, ou bien se focaliser sur des bénéfices consommateurs globaux n'empiétant pas sur l'image des canaux ?

Comme le laisse présager le travail empirique d'Helgesen et al. (2010), il est probable que l'avènement de la distribution multicanal, impliquant de prendre aussi en compte l'image du site marchand, rendra indispensable de mieux discerner ce qui est propre à l'enseigne, à son réseau de magasins, à sa boutique en ligne ou aux produits qu'elle commercialise sous sa marque. Ces recherches pourraient aussi examiner les relations existant entre ces différents concepts, Helgesen et al. ayant fait l'hypothèse d'une influence de l'image de l'enseigne sur celle des points de vente, et non pas l'inverse, hypothèse qui serait tout aussi justifiée, une bonne image du magasin pouvant contrebalancer une mauvaise image de l'enseigne (Gupta et Pirsch, 2008).

\section{Bibliographie}

Aaker J. (1997), «Dimensions of brand personality », Journal of Marketing Research, Vol. 34, No. 3, p. 347-356.

Ailawadi K. et Keller K. (2004), «Understanding retail branding: conceptual insights and research priorities », Journal of Retailing, Vol. 80, p. 331-342.

Ambroise L., Ferrandi J-M., Valette-Florence P. et Merunka D. (2003), "Première application du baromètre de mesure de la personnalité de la marque à deux enseignes

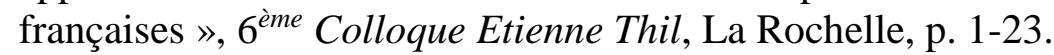

Andrieu F., Badot O. et Macé S. (2004), «Le West Edmonton Mall : un échafaudage sensoriel au service d'une cosmogonie populaire ? », Revue Française de Marketing, No. 196, p. 53-66.

Antéblian-Lambrey B. et François G. (1998), «Une investigation empirique des attitudes, comportements et perceptions envers les produits et les points de vente », Colloque Etienne Thil, La Rochelle, p. 1-15.

Arnold S., Ma S. et Tigert D. (1978), « A comparative analysis of determinant attributes in retail store selection », Advances in Consumer Research, Vol. 5, No. 1, p. 663-667.

Aurier P., Evrard Y. et N'Goala G. (2000), «Valeur de consommation et valeur globale : une application au cas de la consommation cinématographique », Congrès International de l'AFM, Montréal, p. 1-12.

Aurier P., Evrard Y. et N'Goala G. (2004), «Comprendre et mesurer la valeur du point de vue du consommateur », Recherche et Applications en Marketing, Vol. 19, No. 3, p. 1-20.

Badot O. (2005), «L'autre raison du succès de Wall-Mart : une rhétorique de l'infraordinaire », Revue Française de Marketing, No. 203, p. 97-117.

Baker J., Grewal D. et Parasuraman A. (1994), " The influence of store environment on quality inferences and store image », Journal of the Academy of Marketing Science, Vol. 22, No. 4, p. 328-39.

Baker J., Parasuraman A., Grewal D et Voss G. (2002), "The influence of multiple store environment cues on perceived merchandise value and patronage intentions », Journal of Marketing, Vol. 66, p. 120-141.

Bell S. (1999), «Image and consumer attraction to intraurban retail areas: an environmental psychology approach », Journal of Retailing and Consumer Services, Vol. 6, p. 67-78.

Berry L. (1969), « The components of department store images: a theoretical and empirical analysis », Journal of Retailing, Vol. 45, No. 1, p. 3-20.

Birtwistle G., Clarke I. et Freathy P. (1999), «Store image in the UK fashion sector: consumer versus retailer perceptions », International Review of Retail, Distribution and Consumer Research, Vol. 9, No. 1, p. 1-16. 
Bitner M. (1992), «Servicescapes: the impact of physical surroundings on customers and employees », Journal of Marketing, Vol. 56, No. 2, p. 57-71.

Bloemer J. et De Ruyter K. (1998), «On the relationship between store image, store satisfaction and store loyalty », European Journal of Marketing, Vol. 32, No. 5-6, p. 499-513. Bouchet P. (2004), «L'expérience au cœur de l'analyse des relations magasin-magasineur », Recherche et Applications en Marketing, Vol. 19, No. 2, p. 53-71.

Bressolles G. et Durieu J. (2008), «L'impact de la qualité de service électronique sur la satisfaction et les intentions de fidélité : différence entre acheteurs et visiteurs », $24^{\text {ème }}$ Congrès International de l'AFM, Paris, p. 15-16 mai.

Bucklin L. (1966), « Testing propensities to shop », Journal of Marketing, Vol. 30, p. 22-27.

Buttle F. (1985), " Measuring food store image using Kelly's repertory grid », Service Industries Journal, Vol. 5, No. 1, p. 79-89.

Carman J. (1990), "Consumer perceptions of service quality: an assessment of the SERVQUAL dimensions », Journal of Retailing, Vol. 66, No. 1, p. 33-55.

Carù A. et Cova B. (2003), "Revisiting consumption experience: a more humble but complete view of the concept", Marketing Theory, Vol. 3, p. 267-286.

Chardon O. et Dumartin S. (1998), «Fidélité aux enseignes, fidélité aux marques: les choix des consommateurs », Insee Première, No. 609, p. 1-4.

Chowdhury J., Reardon J. et Srivastava R. (1998), «Alternative modes of measuring: an empirical assessment of structured versus unstructured measures », Journal of Marketing Theory and Practice, Vol. 6, No. 2, p. 72-86.

Cohen J. et Basu K. (1987), "Alternative models of categorization: toward a contingent processing framework », Journal of Consumer Research, Vol. 13, p. 455-472.

Collins-Dodd C. et Lindley T. (2003), «Store brands and retail differentiation: the influence of store image and store brand attitude on store own brand perceptions », Journal of Retailing \& Consumer Services, Vol. 10, p. 345-352.

Colombel L., Savard M. et Perrien J. (2007), « Processus de recherche d'information dans le domaine des services : choix et utilisation des canaux d'acquisition de cette information », $12^{\text {èmes }}$ journées de recherche en Bourgogne, p. 1-16.

Coutelle P. (1998), «L'image du point de vente : la formation de prior attitudes », Journées de Recherche en Marketing de Bourgogne, p. 144-161.

Csikszentmihalyi M. (1997), «Finding flow », Psychology Today, Vol. 30, 4, p. 46-71

D’Astous A., Hadj Said I. et Lévesque M. (2002), "Conception et test d'une échelle de

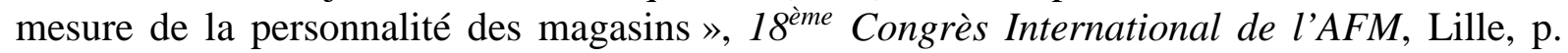
115-130.

Dichter E. (1985), «What's in an image », Journal of Consumer Marketing, Vol. 2, No. 1, p. 75-79.

Dick A., Chakravarti D. et Biehal G. (1990), « Memory-based inferences during consumer choice », Journal of Consumer Research, Vol. 17, p. 82-93.

Donovan R. et Rossiter J. (1982), «Store atmosphere: an environmental psychology approach», Journal of Retailing, Vol. 58, No. 1, p. 34-57.

Donovan R., Rossiter J., Marcoolyn G. et Nesdale A. (1994), «Store atmosphere and purchasing behaviour », Journal of Retailing, Vol. 70, No. 3, p. 283-294.

Doyle P. et Fenwick I. (1974), « How store image affects shopping habits in grocery chains », Journal of Retailing, Vol. 50, No. 4, p. 39-52.

Dubois P-L. (1980), «Le concept de relief des attributs », Revue Française de Marketing, Vol. 81, p. 19- 30.

Duncan C. et Oshavsky R. (1982), «External search: the role of consumer beliefs », Journal of Marketing Research, Vol. 19, p. 32-43. 
Enis B. (1967), «An analytical approach to the concept of image », California Management Review, Vol. 9, No. 4, p. 51-58.

Eroglu S., Machleit K. et Davis L. (2001), «Atmospheric qualities of online retailing: a conceptual model and implications », Journal of Business Research, Vol. 54, p. 177-184.

Ettis S. (2005), «L'atmosphère des sites web marchand : impact de la couleur, des animations et de la musique sur les réponses du consommateur », Tutorat ALM Toulouse, p. 1-56.

Filser M. (2004), «La stratégie de la distribution : des interrogations managériales aux contributions académiques », Revue Française de Marketing, Vol. 198, No. 3/5, p. 7-17.

Finn D. et Lamb C. (1991), "An evaluation of the SERVQUAL scales in a retailing setting », Advances in Consumer Research, Vol. 18, p. 483-489.

Gharbi J-E., Ettis S. et Ben Mimoun M. (2002), «Impact de l'atmosphère perçue des sites

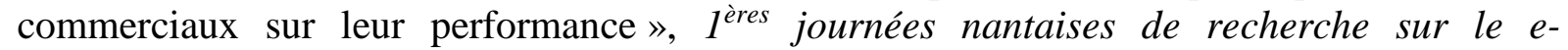
marketing, p. 181-219.

Gaur S. et Agrawal R. (2006), «Service quality measurement in retail store context: a review of advances made using SERVQUAL and RSQS», The Marketing Review, Vol. 6, p. 317-330. Gautschi D. (1981), «Specification of patronage models for retail center choice», Journal of Marketing Research, Vol. 18, p. 162-74.

Gavard-Perret M-L. (1987), «L'image : supériorité et limites. Relation avec l'imagerie mentale et le langage verbal », Recherche et Applications en Marketing, Vol. 11, No. 2, p. 4980 .

Gentry J. et Burns A. (1977), «How "important" are evaluative criteria in shopping center patronage? », Journal of Retailing, Vol. 53, No. 4, p. 73-95.

Golden L., Albaum G. et Zimmer M. (1987), "The numerical comparative scale: an economical format for retail image measurement », Journal of Retailing, Vol. 63, No. 4, p. 393-407.

Grewal D., Krishnan R., Baker J. et Borin N. (1998), «The effect of store name, brand name and price discounts on consumers' evaluations and purchase intentions », Journal of Retailing, Vol. 74, No. 3, p. 331-352.

Gupta S. et Pirsch J. (2008), "The influence of a retailer's corporate social responsibility program on re-conceptualizing store image », Journal of Retailing and Consumer Services, Vol. 15, p. 516- 526.

Gurviez P. et Korchia M. (2002), «Proposition d'une échelle de mesure multidimensionnelle de la confiance dans la marque », Recherche et Applications en Marketing, Vol. 17, No. 3, p. 41-61.

Hansen R. et Deutscher T. (1977), «An empirical investigation of attribute importance in retail store selection », Journal of Retailing, Vol. 53, No. 4, p. 59-95.

Hartman K. et Spiro R. (2005), «Recapturing store image in customer-based store equity: a construct conceptualization », Journal of Business Research, Vol. 58, p. 1112-1120.

Helgesen O., Havold J. et Nesset E. (2010), «Impacts of store and chain images on the "quality-satisfaction-loyalty process", in petrol retailing », Journal of Retailing and Consumer Services, Vol. 17, p. 109-118.

Hetzel P. (2000), «Les approches socio-sémiotiques du design d'environnement des lieux de distribution postmodernes » in P. Volle (cood.), Etudes et recherches sur la distribution, Paris, Economica.

Hildebrandt L. (1988), «Store image and the prediction of performance in retailing », Journal of Business Research, Vol. 17, p. 91-100.

Hirschman E., Greenberg B. et Robertson D. (1978), « The intermarket reliability of retail image research: an empirical examination », Journal of Retailing, Vol. 54, No. 1, p. 3-12. 
Hsu M., Huang Y. et Swanson S. (2010), « Grocery store image, travel distance, satisfaction and behavioral intentions ", International Journal of Retail \& Distribution Management, Vol. 38, No. 2, p. 115-132.

Huber J. et McCann J. (1982), "The impact of inferential beliefs on product evaluations », Journal of Marketing Research, Vol. 19, p. 324-333.

Hui M. et Bateson J. (1991), «Perceived control and the effects of crowding and consumer choice on the service experience », Journal of Consumer Research, Vol. 18, p. 174-184.

Huvé-Nabec L. (1998), «L'évaluation des extensions du circuit de distribution par le consommateur : le rôle des représentations respectives de la marque et de l'enseigne dans la mémoire du consommateur », $3^{\text {èmes }}$ Journées de Recherche en Marketing de Bourgogne, $\mathrm{p}$. 89-106.

Huvé-Nabec L. (2002), «L'évolution des représentations de la marque et de l'enseigne dans

l'esprit du consommateur lors de la formation de nouveaux couples marque-enseigne », $18^{\text {ème }}$ Congrès International de l'Association Française du Marketing, p. 601-632.

Ingarao A. (2004), «L'image en marketing: revue de littérature d'un concept multidimensionnel », Cahier de Recherche Cermat, Vol. 17, No. 04-113, p. 43-67.

Jain A. et Etgar M. (1976), «Measuring store image through multidimensional scaling of free response data », Journal of Retailing, Vol. 52, No. 4, p. 61-96.

Jallais J., Orsoni J. et Fady A. (1987), Marketing de la distribution, Vuibert Gestion.

Jallais J., Orsoni J. et Fady A. (1994), Le marketing dans le commerce de détail, Paris, Vuibert.

James D., Durand R. et Dreves R. (1976), «The use of a multi-attribute model in a store image study », Journal of Retailing, Vol. 52, No. 2, p. 23-32.

Jara M. (2006), «Nouvelle proposition conceptuelle du capital-marque : une approche par l'enseigne et le point de vente », www.reseau-iae.org/tutorats/tutorat12.pdf, p. 1-18.

Joyce M. et Lambert D. (1996), « Memories of the way stores were and retail store image », International Journal of Retail \& Distribution Management, Vol. 24, No. 1, p. 24-33.

Keaveney S. et Hunt K. (1992), «Conceptualization and operationalization of retail store image: a case of rival middle-level theories», Journal of the Academy of Marketing Science, Vol. 20, No. 2, p. 165-175.

Keller K. (1993), « Conceptualizing, measuring, and managing customer-based brand equity », Journal of Marketing, Vol. 57, p. 1-22.

Kelly P. et Smith S. (1983), «Determinants of retail store patronage: an examination of alternative models », Advances in Consumer Research, Vol. 10, No. 1, p. 345-350.

Kerin R., Jain A. et Howard D. (1992), «Store shopping experience and consumer pricequality-value perceptions », Journal of Retailing, Vol. 68, No. 4, p.376-397.

Kirmani A. et Rao A. (2000), «No pain, no gain: a critical review of the literature on signalling unobservable product quality », Journal of Marketing, Vol. 64, p. 66-79.

Kotler P. (1973), «Atmospherics as a marketing tool », Journal of Retailing, Vol. 49, No. 4, p. 48-64.

Kunkel J. et Berry L. (1968), "A behavioral conception of retail image », Journal of Marketing, 32, 21-27.

Ladwein R. (1998), «Stratégies de marques et concepts de marques », Décisions Marketing, Vol. 13, p. 17-24.

Lazer W. et Wyckham R. (1969), «Perceptual segmentation of department store markets », Journal of Retailing, Vol. 45, No. 2, p. 3-14.

Lemoine J-F. et Badot O. (2008), «Gestion tribale de la marque et distribution spécialisée : le cas Abercrombie \& Fitch », Décisions Marketing, No. 52, p. 9-18.

Lessig P. (1973), «Consumer store images and store loyalties », Journal of Marketing, Vol. 37, No. 4, p. 72-74. 
Lindquist J.D. (1974), « Meaning of image », Journal of Retailing, Vol. 50, No. 4, p. 29-38.

Loiacono E., Watson R. et Goodhue D. (2007), «WebQual: an instrument for consumer evaluation of web sites », International Journal of Electronic Commerce, Vol. 11, No. 3, p. 51-87.

Louis D. et Pederzoli D. (2005), «La personnalité de la marque : application au domaine de la distribution et influence sur l'image de l'enseigne », $4^{\text {èmes }}$ Journées Normandes de Recherches sur la Consommation, Rouen, p. 1-25.

Louis D., Lombart C. et Pederzoli (2006), «The image of retailers in France », European Regional Review, Vol. 51, p. 26-31.

Malhotra N. (1983), « A threshold model of store choice, Journal of Retailing, Vol. 59, No. 2, p. 3-21.

Marion G. (1989), Les images de l'entreprise, Edition Organisation.

Martineau P. (1958), «The personality of the retail store », Harvard Business Review, Vol. 36, No. 1, p. 47-55.

Mathwick C., Malhotra N. et Rigdon E. (2001), «Experiential value: conceptualization, measurement and application in the catalog and Internet shopping environment», Journal of Retailing, Vol. 77, No. 1, p. 39-56.

Mazursky D. et Jacoby J. (1986), «Exploring the development of store images », Journal of Retailing, Vol. 62, No. 2, p. 145-165.

Michon R., Chebat J-C. et Turley L. (2005), «Mall atmospherics: the interaction effects of the mall environment on shopping behavior », Journal of Business Research, Vol. 58, p. 576583.

Mitchell W. (1984), «What is an image? », New Literary History, Vol. 15, No. 3, p. 503-537.

Morschett D., Swoboda B. et Foscht T. (2005), «Perception of store attributes and overall attitude towards grocery retailers: the role of shopping motives », International Review of Retail, Distribution and Consumer Research, Vol. 15, No. 4, p. 423-447.

Myers J. et Alpert M. (1977), «Semantic confusion in attitude research: salience vs. importance vs. determinance », Advances in Consumer Research, Vol. 04, p. 106-110.

N'Gobo P-V. (2006), «Entretien : l'image comme déterminant du choix d'un magasin et du panier moyen », La lettre Marketing Scan, Vol. 31, p. 5-6.

Nevin J. et Houston M. (1980), «Image as a component of attraction to intra-urban shopping areas », Journal of Retailing, Vol. 56, No. 1, p. 77-93.

Nicholson P. et Vanheems R. (1998), «Les stratégies de multi-implantation des enseignes : l'influence de l'extension d'un réseau de vente sur la perception et la fréquentation des points de vente », $3^{\text {èmes }}$ Journées de Recherche en Marketing de Bourgogne, Dijon, p. 107-117.

Oppewal H. et Timmermans H. (1997), «Retailer self-perceived store image and competitive position », International Review of Retail, Distribution and Consumer Research, Vol 7, No. 1, p. 41-59.

Osman M. (1993), «A conceptual model of retail image influences on loyalty patronage behaviour », International Review of Retail, Distribution \& Consumer Research, Vol. 3, No. 2, p. 133-148.

Oxenfeldt A. (1974), «Developing a favorable price-quality image », Journal of Retailing, Vol. 50, No. 4, p. 8-14.

Paivio A. (1991), « Dual coding theory: retrospect and current status », Canadian Journal of Psychology, Vol. 43, No. 3, p. 255-287.

Pan Y. et Zinkhan G. (2006), " Determinants of retail patronage: a meta analytical perspective», Journal of Retailing, Vol. 82, p. 229-243.

Pessemier E. (1980), «Store image and positioning », Journal of Retailing, Vol. 56, No. 1, p. 94-106. 
Pontier S. (1988), «Image du point de vente : pour une prise en compte de l'image interne », Recherche et Applications en Marketing, Vol. 3, No. 3, p. 3-19.

Purohit D. et Srivastava J. (2001), «Effect of manufacturer reputation, retailer reputation, and product warranty on consumer judgments of product quality: a cue diagnosticity framework », Journal of Consumer Psychology, Vol. 10, No. 3, p. 123-134.

Rattier M. (2002), «L'image en marketing: cadre théorique d'un concept multidimensionnel », Cahier de Recherche IAE Toulouse, Vol. 152, p. 1-21.

Reeves C. et Bednar D. (1994), «Defining quality: alternatives and implications », Academy of Management Review, Vol. 19, No. 3, p. 419-445.

Roederer C. (2008), «L'expérience de consommation: exploration conceptuelle, méthodologique et stratégique », Thèse de Doctorat en Sciences de Gestion, Université de Bourgogne, Dijon.

Rolland S. et Freeman I. (2010), "A new measure of e-service quality in France », International Journal of Retail \& Distribution Management, Vol. 38, No. 7, p. 497-517.

Russell J. et Mehrabian A. (1976), «Environmental variables in consumer research », Journal of Consumer Research, Vol. 3, No. 1, p. 62-63.

Samli C., Kelly P. et Hunt K. (1998), «Improving the retail performance by contrasting management and customer perceived store images: a diagnostic tool for corrective action », Journal of Business Research, Vol. 43, No. 1, p. 27-38.

Schiffman L., Dash J. et Dillon W. (1977), «The contribution of store-image characteristics to stereotype choice », Journal of Retailing, vol. 53, No. 2, p. 3-16.

Semeijn J., Van Riel A. et Ambrosini A. (2004), "Consumer evaluations of store brands: effects of store image and product attributes », Journal of Retailing and Consumer Services, Vol. 11, No. 4, p. 247-258.

Stanley T. et Sewall M. (1976), «Image inputs to a probabilistic model: predicting retail potential », Journal of Marketing, Vol. 40, p.48-53.

Steenkamp J-B. et Wedel M. (1991), «Segmenting retail markets on store image using a consumer-based methodology », Journal of Retailing, Vol. 67, No. 3, p. 300-320.

Tarafdar M. et Zhang J. (2007), «Determinants of reach and loyalty - a study of website performance and implications for website design», Journal of Computer Information Systems, Vol. 48, No. 2, p. 16-24.

Turley L. et Milliman R. (2000), «Atmospheric effects on shopping behaviour: a review of the experimental evidence », Journal of Business Research, Vol. 49, p. 193-211.

Wang S., Beatty S. et Mothersbaugh D. (2009), "Congruity's role in website attitude formation », Journal of Business Research, Vol. 62, No. 6, p. 609-615.

Ward J., Bitner M. et Barnes J. (1992), « Measuring the prototypicality and meaning of retail environments », Journal of retailing, Vol. 68, No. 2, p. 194-219.

Wu B. et Petroshius S. (1987), «The halo effect in store image measurement », Journal of the Academy of Marketing Science, Vol. 15, No. 3, p. 44-51.

Zeithaml V. (1988), «Consumer perceptions of price, quality and value: a means-end model and synthesis of evidence », Journal of Marketing, Vol. 52, p. 2-22.

Zimmer M. et Golden L. (1988), «Impressions of retail stores: a content analysis of consumer images », Journal of Retailing, Vol. 64, No. 3, p. 265-293. 